\title{
Gene therapy progress and prospects: the skin - easily accessible, but still far away
}

\author{
UR Hengge \\ Department of Dermatology, Heinrich-Heine-University, Duesseldorf, Germany
}

\begin{abstract}
Significant progress has been made in corrective gene therapy of inherited skin diseases. This includes advances in vector technology, targeted gene expression, gene replacement, and the availability of appropriate animal models for a variety of candidate diseases. In addition, an increased understanding of the uptake and trafficking mechanisms inside keratinocytes has evolved. Topical application facil-
\end{abstract}

itates DNA vaccination through the skin, albeit clinical benefits have not yet materialized. However, the translation into clinical trials has only been partially mastered. The latter and the control of immune responses represent challenges for the research community.

Gene Therapy (2006) 13, 1555-1563. doi:10.1038/

sj.gt.3302855; published online 7 September 2006

Keywords: inherited blistering skin diseases; skin gene therapy; DNA vaccination; internalization pathways; trafficking; immune response

\section{In brief}

\section{Progress}

- Systematic knowledge on genetic defects of candidate diseases has accumulated.

- Targeted gene expression to the epidermis has been achieved, but was neither a prerequisite for correction nor did it improve longevity of expression.

- Stable integration of large therapeutic genes has been achieved using the sleeping beauty transposon or the ФC31 bacterial phage integrase.

- New technological advances have permitted gradual improvements in gene silencing and gene repair.

- Suitable animal models have demonstrated proof of concept for treating human genodermatoses.

- Preclinical progress has been achieved in the treatment of wounds, epidermolysis bullosa and ichthyosis.

- Clinical vaccination trials against melanoma have yielded mixed results.

- Undesired immune responses limit duration of gene expression.

- DNA vaccines have largely disappointed in the human setting and need refinement.

- Topical spray application of liposomal DNA enables vaccination.

- Mechanisms of DNA uptake and trafficking reveal fundamental insights into keratinocyte biology.

\section{Prospects}

- The correction of a certain percentage of defective keratinocytes or fibroblasts may prove sufficient for functionality of the neo-skin to occur.

- Supplementation therapy of genes with similar, but independent functions (e.g. desmin for keratin) may alleviate the effect of dominant-negative proteins.

- Direct in vivo technologies for gene transfer/ correction will gradually replace the ex vivo gene transfer protocols for treating larger skin areas; ex vivo strategies will probably not make it into larger trials.

- Dominant-negative diseases will become treatable by inactivation of the mutant protein by RNAi technology or zinc-finger nucleases.

- Large animal models are desirable to determine the safety and efficacy of in vivo therapies before testing in human subjects.

- Undesired immune responses against neo-antigens will lead to immunosuppressive treatment protocols around the time of gene transfer to permit extended gene expression.
Correspondence: Professor UR Hengge, Department of Dermatology, Heinrich-Heine-University, Moorenstrasse 5, D-40225 Duesseldorf, Germany.

E-mail: ulrich.hengge@uni-duesseldorf.de

Received 29 November 2005; revised 12 July 2006; accepted 22 July 2006; published online 7 September 2006

\section{Introduction}

As the skin is the largest and most accessible organ, it provides an attractive target for therapeutic gene transfer. Skin is composed of the self-renewing epithelial layers, called epidermis, the dermis and the subcutaneous fat layer (Figure 1). While the epidermis contains the most abundant epithelial cells (i.e. keratinocytes), melanocytes (leading to pigmentation), and Langerhans 


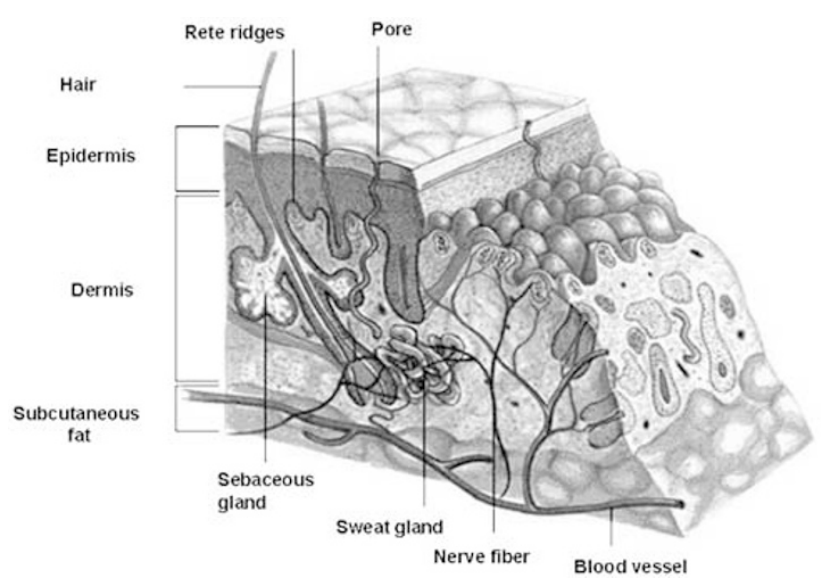

Figure 1 Structure and components of the skin.

cells (i.e. epidermal dendritic cells), it is not vascularized. Therefore, proteins synthesized in the epidermis have to cross the basement membrane, which anchors the epidermis to the dermis prior to gaining access to the bloodstream. Keratinocytes stem cells divide in the basal portion of the epidermis and the bulge area of the hair follicle to give rise to transient amplifying cells that undergo layer-specific differentiation regulated by specific promoters (e.g. keratin 5 and 14 in the basal layer, keratin 1 and 10 in the granular layer, and involucrin and transglutaminase in the uppermost layers, respectively). Ultimately the horny layer (strateum corneum) protects the skin from the environment (e.g. from evaporation, pathogens and allergens etc.) and forms the tight epidermal barrier that has to be overcome, for topical skin gene therapy to become a reality. The epidermal turnover takes about 28 days and therefore limits longterm expression, as transient amplifying cells are preferentially transfected. The components of the basement membrane zone may be mutated and cause inherited blistering diseases that represent a major target for gene therapy. The dermis contains the blood vessels, hair follicles, sweat and sebaceous gland embedded in a delicate network of interstitial matrix (collagen, elastic fibers, glycosaminoglycans) secreted by fibroblasts.

Generally, genes can be transferred to the skin by both in vivo- and ex vivo-strategies (Figure 2). Since the last comprehensive review of the field, a number of new achievement has been made in modifying skin cells for gene therapy purposes.

\section{Systematic knowledge on genetic defects of candidate diseases has accumulated}

Given molecular genetics that has tremendously improved the understanding of genotype-phenotype correlations in cutaneous genodermatoses (Uitto and Richard ${ }^{1}$; Figure 3), gene therapy has begun to achieve functional correction. For example, deficient gene expression of type VII-collagen (one of the anchoring fibers usually produced by fibroblasts) has been successfully corrected - at least for 16 weeks - by in vitro cotransfection with a $\phi \mathrm{C} 31$ integrase-encoding plasmid and a type VII-collagen plasmid of fibroblasts from patients with recessive dystrophic epidermolysis bullosa

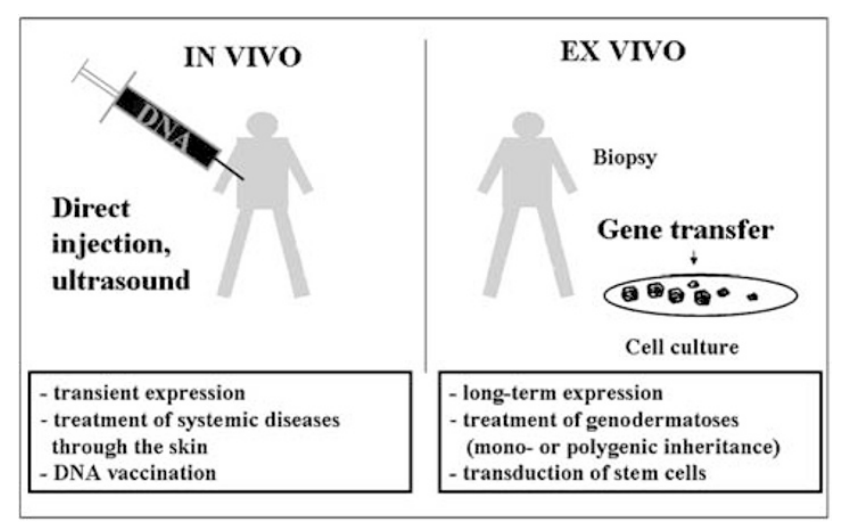

Figure 2 Ex vivo versus in vivo strategy. The principle of gene transfer with regard to the skin is depicted. Hallmarks of both strategies are listed.

(EB) followed by injection into recessive dystrophic EB skin. Interestingly, synthesized type-VII-collagen was found to correctly localize upon reconstitution of a neo-skin and several hallmarks of EB, including subepidermal blistering and anchoring fiber defects have normalized. ${ }^{2}$

In principle, the injection of genetically engineered fibroblasts into the dermis offers a number of advantages over epidermal grafting strategies. Fibroblasts are (i) more robust cells than keratinocytes, and (ii) much less susceptible to differentiation than epidermal progenitors. Moreover, unlike epidermal sheets, that are fragile and must be immediately used, (iii) fibroblasts can be delivered to intact skin via intradermal injection of single cell suspensions, eliminating the need for subsequent wound care.

In contrast, the initial studies, using epidermal sheets derived from gene-corrected keratinocytes, have achieved correction at the histological, molecular and functional level, but failed to extend beyond 2-4 weeks, which is the estimated time needed for epidermal turnover. Even when the longevity of the collagen gene product is considered, correction may not prove beneficial for patients long-term using this vector system.

\section{Targeted gene expression to the epidermis has been achieved, but was neither a prerequisite for correction nor did it improve longevity of expression}

The compartmentalization of epidermis and its continuous renewal challenge vectors for targeted transgene expression. On one hand, long-term expression should become possible if gene transfer to progenitor (stem) cells in the basal layer was successful. However, keratin 14 promotors still resulted in gene expression in both basal keratinocytes and their suprabasal (i.e. differentiating) progeny, with gene expression being gradually lost due to desquamation and promoter inactivation (for review see Hengge). ${ }^{3}$

On the other hand, stratum-specific expression to suprabasal (i.e. differentiating) layers was not required in lamellar ichthyosis, a monogenic skin disorder of transglutaminase, to achieve morphological correction. 


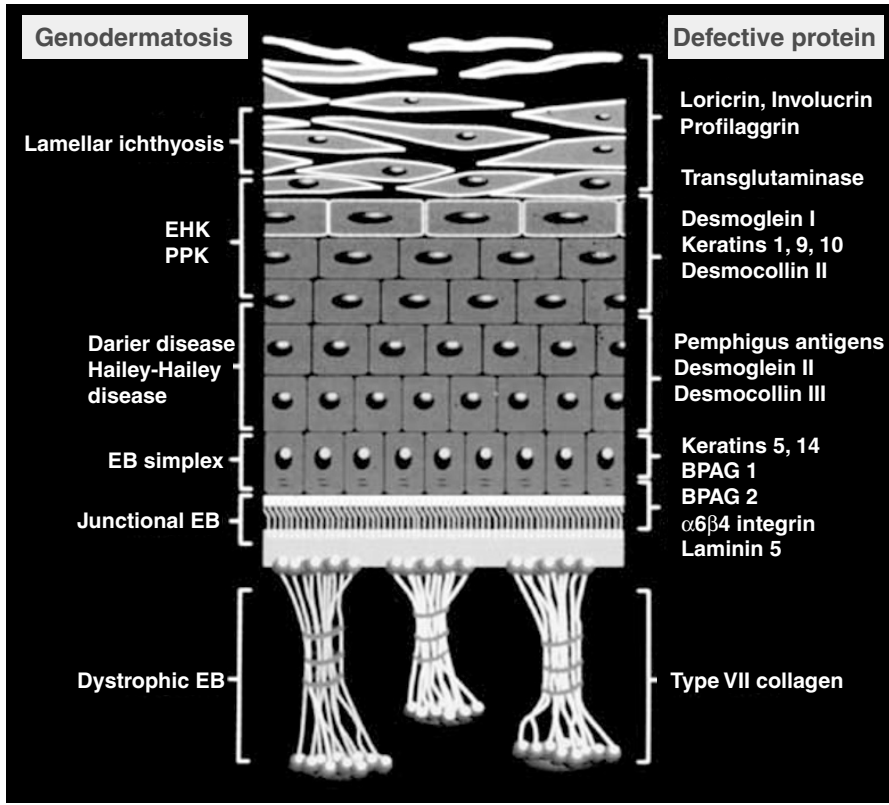

Differentiation

Epidermis

Basal cell membrane

Dermis

Figure 3 Summary of genodermatoses. Shown are the epidermis, the basement membrane and the papillary dermis. On the left side the genetic diseases are mentioned with the protein defects detailed on the right side. EHK, epidermolytic hyperkeratosis; PPK, palmoplantar keratosis; EB, epidermolysis bullosa; BPAG, bullous pemphigoid antigen.

Again, expression of the therapeutic gene was lost over a period of several weeks, despite the fact that immunodeficient animals were used (for review see Hengge). ${ }^{3}$ Besides gene inactivation, a selective growth disadvantage for transduced stem cells and a gradual loss of these cells from the treated tissue have been proposed.

As integrating viral vectors probably have the best chance of enabling long-term expression, immortalized keratinocytes and primary keratinocytes were transduced by a variety of viral vectors and analyzed for long-term expression. ${ }^{4,5}$ Not surprisingly, recombinant retroviruses yielded sustained levels of transgene expression, but lentiviral vectors showed the highest ability to transduce clonogenic primary keratinocytes. ${ }^{4}$ When cells were cultured on a substrate of recombinant fibronectin, preferential transduction of early precursor stem cells could be achieved. ${ }^{6}$

Efficient gene delivery into keratinocytes has also been demonstrated for adeno-associated virus type-2 (rAAV2). ${ }^{7}$ However, other investigators were unable to transduce primary keratinocytes with AAV vectors. ${ }^{5}$ Impaired cellular trafficking of vector particles and high levels of autophosphorylation of the epidermal growth factor receptor (EGF-R) tyrosine kinase present impediments to gene transfer into keratinocytes. To improve the efficiency of gene delivery, treatment of keratinocytes with proteasome inhibitors increased the percentage of transduced cells by $33 \%$ and prolonged the duration of expression until 10 weeks, at which point senescence was reached. ${ }^{7}$

Aside from cellular targeting, transcriptional targeting has recently also been assessed in primary melanoma cells, the most important skin tumor. ${ }^{8}$ In these experiments, the survivin promotor exhibited the highest activity in melanoma cells, while normal tissues such as liver, lung and spleen were relatively spared. Combinations of cellular targeting using RGD fiber-mutant adenovirus vectors that contain an alpha-v integrin tropism with the melanoma-specific tyrosinase promotor or human telomerase reverse transcriptase (TERT) promotor have shown increased gene expression in tumor cells with a relative quiescence in normal cells. ${ }^{9}$

\section{Stable integration of large therapeutic genes has been achieved using the sleeping beauty transposon or the $\Phi \mathrm{C} 31$ bacterial phage integrase}

Initial approaches to phenotypic reversion of EB have relied on grafting engineered epidermal sheet tissue. As a number of drawbacks (e.g. costs, fragility of engineered epidermal tissue and removal of host tissue before grafting) limits its application in humans and since direct administration of viral vectors is limited by efficiency, cell targeting and biosafety, the sleeping beauty (SB) transposable element was used as an alternative to integrate incoming DNA into the genome using short inverted repeat sequences flanking the insert and the SB transposase protein, resulting in long-term gene expression. This technology has been successfully used to integrate the LAMB3 cDNA into epidermal holoclones from unrelated junctional EB patients. ${ }^{10}$ Epidermal holoclones have the greatest reproductive capacity and represent $<5 \%$ of primary keratinocyte suspensions; they are considered to contain true epidermal stem cells. Non-viral integration of the LAMB3 gene led to normalized levels of laminin-5 protein, hemidesmosome formation and a normal phenotype. ${ }^{10}$

Alternatively, the ФC31 bacterial phage integrase, which inserts - albeit with low efficiency-, large genes into genomes was used to stably integrated the type-VII collagen gene into primary epidermal progenitor cells of 
four unrelated recessive dystrophic EB patients. ${ }^{11}$ Again, collagen-VII found its correct location in regenerated epidermis and located to the basement membrane zone. With the ability to transfer large therapeutic genes, which cannot be packaged in viral vectors, into the genome of patients a significant limitation of cutaneous gene therapy has been overcome. However, - at present the efficiency of transfer and the longevity of expression are probably too low to achieve meaningful effects.

\section{New technological advances have permitted gradual improvements in gene silencing and gene repair}

Dominant disorders cannot be corrected by simply overexpressing the correct protein. Recently, short inhibitory RNA (RNAi) technology has been developed that may be adapted to deactivate dominant-negative alleles. ${ }^{12}$ The silencing of dominant-negative proteins would permit the functional effect of newly introduced wild-type genes for example in dominant forms of EB. Alternatively, gene repair has been successful by specifically designed zinc-finger nucleases using homologous recombination. ${ }^{13}$ The clinical benefit of both strategies still remains to be demonstrated.

An alternative strategy of treating dominant disorders has recently been proposed. The so-called supplementation therapy aims to bypass the defective gene by inducing the expression of another gene with similar function, but which is not susceptible to the disruptive effect of the mutant. In a recent example, desmin, an intermediate filament protein normally expressed in muscle cells, has been transfected into keratinocytes from patients with EB simplex harboring a dominantnegative mutation in keratins $\mathrm{K} 5$ or K14. ${ }^{14}$ Such supplementation therapy transiently restored responses to stress such as scratch wounding, heat or osmotic shock. ${ }^{14}$ This treatment is more universal and amendable to a larger cohort of patients with related individual mutations.

\section{Suitable animal models have demonstrated proof of concept for treating human genodermatoses}

An essential requirement in the development of any gene therapy is the availability of inducible animal models of genetic diseases. As universal expression of the mutant genes of interest is lethal, small test areas of genetically engineered mouse skin are induced to express the mutation by application of a chemical compound. This conditional expression is reversible. Recently, two inducible mouse models of keratin disorders have been developed. ${ }^{15}$ In the models of epidermolysis bullosa simplex and bullous congenital ichthyosiform erythroderma $(E H K)$, the mutant gene is induced upon topical application of a chemical agent. Appropriate, larger animal models are required to determine the safety and efficacy of novel in vivo therapies before testing in human subjects.

A spontaneously occurring large animal model has been identified as the recessive dystrophic EB in golden retrievers where type-VII collagen is absent. This disease has successfully been corrected using retroviral vectors and ex vivo gene transfer. ${ }^{16}$ Skin equivalents obtained from recessive dystrophic EB fibroblasts and corrected keratinocytes showed normal structural and functional effects. ${ }^{17}$ On a similar note, the intradermal injection of lentiviral vectors into human dystrophic EB composite skin equivalents grafted onto immunodeficient mice has been investigated. ${ }^{18}$ The lentiviral vectors containing the type-VII collagen gene transduced dermal fibroblasts, which exported the type-VII collagen into the basement membrane zone, where it formed anchoring fiber structures. ${ }^{18}$ While a single lentiviral vector injection provided significant functional improvement for at least 3 months in this mouse model of human dystrophic EB, the therapy remained confined to the injection site.

Patients with the autosomal recessive DNA repair disorder xeroderma pigmentosum (XP) suffer from a high incidence of skin cancer following UV exposure. Recombinant adenovirus encoding the human XPA gene was used for in vivo gene therapy in UVB-irradiated skin of XP mutant mice. Subcutaneous adenovirus injection led to the expression of the XPA protein in basal keratinocytes and prevented deleterious effects including the development of squamous cell carcinoma. ${ }^{19}$ This therapy may hold promise for preventing skin cancers in the areas most severely exposed to UV light in patients with XP.

Graft necrosis represents a significant hurdle to large area skin transplantation. When vascular endothelial growth factor (VEGF) plasmid DNA was injected in the subcutaneous fascial layers of the upper abdominal wall in a rat pedicled TRAM flap model, significantly more vessel sprouting led to increased graft survival. ${ }^{20}$

\section{Preclinical progress has been achieved in the treatment of wounds, epidermolysis bullosa and ichthyosis}

As infection represents the major problem in severely burned patients causing skin graft failure and given the emerging resistance of antibiotic-resistant microorganisms, antimicrobial peptide genes have been delivered to the skin by adenoviral vectors. ${ }^{21}$ This form of skin gene therapy only requires transient expression until the wound has closed. It was shown to significantly inhibit bacterial growth on the wounds. ${ }^{21}$ In a rat model, skin wounded by thermal injury has been transfected using human cathelicidin and proven significantly more effective than the administration of the synthetic host defense peptide. ${ }^{22}$ A similar study using a human $\beta$-defensin-3 (HBD-3) plasmid construct has demonstrated significant antimicrobial activity in patients when transfected skin ulcers were analyzed. ${ }^{23}$ Biopsies from transplanted epidermal sheets showed 2.5-times higher levels of HBD-3 transcripts than those of control skin. ${ }^{23}$

In mouse models of wound healing, electroporation has been successfully introduced to enhance transfection. In a recent study, keratinocyte growth factor (KGF) has been delivered to an excisional wound model via naked DNA injection with subsequent electroporation. ${ }^{24}$ This study reported closure of excisional wounds in diabetic mice in over $90 \%$ of wounds. The untreated group 
achieved wound closure in $40 \%$ by day $12 ;{ }^{24}$ however, no DNA injection only group was included. Similar improvements with regard to increased reepithelialization, collagen synthesis and angiogenesis have also been reported for transforming growth factor- $\beta$ (TGF- $\beta$ )- $1 .{ }^{25}$

Transient skin gene therapy may also hold promise for the inhibition of extensive scar formation. When adenovirus-mediated transfer of truncated TGF- $\beta$-receptor-II was performed in rat wounds, dermal fibroblasts were found to express the truncated receptor. ${ }^{26}$ Interestingly, the expression of TGF- $\beta$ - 1 was markedly downregulated in truncated TGF- $\beta$-receptor-II transduced fibroblasts, leading to decreased scarring upon wound closure. ${ }^{26}$

Another potential candidate disease for skin gene therapy is harlequin ichthyosis, a devastating skin disorder with abnormal lamellar granules in keratinocytes. In a recent publication, the gene defect has been mapped to an ATP-binding cassette transporter family (ABCA) that is involved in lipid secretion via lamellar granules. ${ }^{27}$ Following gene transfer of the ABCA12 cDNA congested lipid secretion could be corrected. ${ }^{27}$

Recent progress has also been reported for gene therapy of human sweat glands serving as a model for cystic fibrosis. ${ }^{28}$ Improved transduction protocols using adenoviral vectors have been described for organ cultured sweat glands upon pretreatment with dispase. ${ }^{28}$

\section{Clinical vaccination trials against melanoma have yielded mixed results}

One dozen trials in patients with metastatic melanoma have been completed in the last couple of years. Most trials have been performed in the area of DNA, RNA or dendritic cell (DC) vaccination. In patients with stage IV melanoma repeated doses of between 2 and $20 \mathrm{mg}$ total DNA encoding the human interleukin-12 (IL-12) gene have been evaluated in nine patients (Heinzerling et al. ${ }^{29}$; Heller et al., personal communication). The authors reported three clinical responses (two stable diseases and one complete remission). All patients except one experienced a transient response at the intratumoral injection site, which correlated with an antigen-specific immune response against melanoma-associated antigens (MAGE-1 and MART-1; Heinzerling et al. ${ }^{29}$ ). Similarly, four intratumoral administrations of a recombinant canarypox virus expressing IL-12 were performed in patients with metastatic melanoma. In this clinical trial involving nine patients inflammatory reactions were reported at the injection site and flu-like symptoms. ${ }^{30}$ In four of nine patients increased circulating levels of IL-12 mRNA and IFN- $\gamma$ mRNA were detected. Treated tumor nodules had increased T-cell infiltration. One patient developed a complete response of the injected subcutaneous metastasis as well as of uninjected in-transit metastases. ${ }^{30}$

A recent phase-I/II trial with autologous melanoma cells modified with the tag-7/PGRP-S gene reported antitumor immune responses in 16 of in 17 melanoma patients. ${ }^{31}$ The Drosophila homologue of the tag-7/PGRP$\mathrm{S}$ gene is known to be involved in the innate immune responses. ${ }^{31}$ However, no clinical responses were seen.

Studies with genetically modified DC were disappointing. DC vaccination with tyrosinase in patients with stage II melanoma using recombinant modified vaccinia virus Ankara has not shown significant immunological or clinical responses. ${ }^{32}$ However, progress has been made in characterizing the CD4+ and CD8+ immune response induced upon DC vaccination of melanoma patients. ${ }^{33}$

\section{Undesired immune responses limit duration of gene expression}

Immunological responses, especially in situations where a new antigen is expressed (i.e. recessive diseases), are likely to limit expression and therapeutic use. It has become increasingly clear that the unique immunological features of the cutaneous microenvironment containing antigen-presenting cells (APCs), including Langerhans cells and dermal DC, along with the secretion of inflammatory cytokines from both keratinocytes and DC are inadvertent conditions for long-term expression of a desired transgene. Previously, it has been shown that in vivo gene transfer leads to the induction of transgenespecific immune responses besides cytotoxic $\mathrm{T}$ cells and antibodies against the vector backbones. The nature of host immune responses following gene transfer depends on various factors, including the immunogenicity of the transgene product and the cell or tissue type producing it. ${ }^{34}$ Moreover, the vector backbone (i.e. virus envelope) is a potential source of non-cell antigens. Whereas longterm expression has been obtained upon retroviral gene transfer in immunodeficient mice, immunocompetent mice were shown to mount a predominantly cellular immune response with transgene-specific cytotoxic $\mathrm{T}$ lymphocytes. ${ }^{34}$ Only in animals deficient in both major histocompatibility complex (MHC) class-I- and class-IImolecules prolonged transgene expression has occurred. In addition, humoral immune responses with IgG predominantly of the $2 \mathrm{a}$ - and $2 \mathrm{~b}$-subtype were detected, but found not to inhibit transgene expression. ${ }^{34}$

Under these circumstances, ex vivo gene transfer may avoid signals provided to the immune system by in vivo administration of vectors. However, despite an ex vivo setting, where primary epidermal cultures were depleted of APCs, specific immune responses against a model transgene were generated and led to the clearance of transduced cells following grafting onto immunocompetent mice. ${ }^{35}$ Only when the immunocompetent mice were tolerant to the indicator protein, permanent engraftment of transduced cells and continued gene expression were achieved. Most likely, upon ex vivo transfer, the activation of transgene-specific immune responses involved crosspresentation of antigens in transduced keratinocytes to $\mathrm{APCs}{ }^{35}$

Additional support for the immunogenicity of neoantigens in hosts with a null mutation of certain proteins came from studies in desmoglein-3 knockout mice that received injections of murine desmoglein-3-cDNA, where $50 \%$ of the injected animals developed antidesmoglein-3-IgG. ${ }^{36}$ To prevent the immune response, an anti-CD40L monoclonal antibody that blocks the co-stimulatory interaction between CD40 and CD40L, significantly prevented the generation of anti-desmoglein-3 antibodies. Alternative methods to overcome the immune response against a transgene product in gene therapy models consist of immunosuppressive therapy around the time of gene delivery. Such immunosuppressive treatment strategies (e.g. cyclophosphamide) have 
been successfully performed in liver-mediated gene therapy for hemophilia. ${ }^{37}$ Immunization using recombinant DNA followed by recombinant modified vaccinia virus Ankara expressing the melanoma antigen mel3 generated specific CTL in the B16 murine melanoma. ${ }^{38}$ Upon combination of this immunotherapeutic regimen with metronomic delivery (i.e. repeated dosing at certain intervals) of cyclophosphamide enhanced antitumor activity was observed when compared with either treatment alone, probably by reducing the numbers of CD4+ and CD25+ regulatory $\mathrm{T}$ cells. ${ }^{38}$ Another study provided evidence that transgene expression following in vivo viral gene transfer can induce CD4+ T-cell tolerance to the transgene product, involving immunologic anergy and deletion. ${ }^{39}$

A recent study investigated, whether immunosuppressive blockade of CTLA-4 could increase T-cell responses and tumor immunity elicited by DNA vaccines. ${ }^{40}$ When mice were immunized against the tyrosinase-related protein-2 and gp100, significantly enhanced B16 melanoma tumor rejection could be achieved when anti-CTLA-4 was administered with booster vaccinations. However, autoimmunity to cellular antigens was also observed. ${ }^{40}$

\section{DNA vaccines have largely disappointed in the human setting and need refinement}

Much in contrast, these undesired immunological features are of paramount importance for genetic vaccination. ${ }^{41}$ To increase their immunogenic potential DNA vaccines have been targeted to epidermal DC by coupling to mannose to facilitate mannose-receptormediated endocytosis leading to improved generation of cytotoxic $\mathrm{T}$ lymphocytes (CTL) and resulting in protection as demonstrated in a rhesus monkey model of HIV infection. ${ }^{42}$ Also, transfection with mRNAs encoding tumor-associated antigens and granulocytemacrophage colony-stimulating factor efficiently primed CTL responses, but was insufficient to overcome tolerance to a model tumor/self antigen. ${ }^{43}$

However, despite presenting relatively few safety concerns, DNA vaccines have been more effective in rodents than in larger animals or humans. Whether this is due to the different adjuvants used in animal and human trials, the stimulation of distinctive subsets of Toll-like receptors that trigger the innate immune system, the different effects on regulatory T-cells, or the different immunogenetic background still needs to be elucidated. Nevertheless, sequential immunization with malaria DNA followed by boosting with recombinant protein has led to the induction of CD8+ and CD4+ T cell and antibody responses in human volunteers. ${ }^{44}$ The CD8+ T cells of the CD45RA (naïve) phenotype seemed to act as effector $\mathrm{T}$ cells. Thus, this immunization strategy has proven the concept of DNA priming and protein boosting in humans. A similar study has confirmed these results by either intramuscular or -epidermal application of a DNA vaccine or recombinant modified vaccinia virus Ankara (MVA) vaccine expressing the thrombospondin-related adhesion protein (a pre-erythrocytic malaria antigen), leading to 5- to 10-fold higher $\mathrm{T}$-cell responses and the secretion of interferon- $\gamma$ than induced by either treatment alone. ${ }^{45}$ Additional strategies have been exploited to increase immunogenicity of vaccines in humans. These strategies integrate in vivo electro- or microporation for improved delivery, ${ }^{46,47}$ the inclusion of various immunostimulatory molecules, xenoantigens or xenoepitopes, antigen-cytokine fusion genes, agents that improve antigen uptake or presentation and immunomodulatory compounds such as CpG motifs. ${ }^{41}$ Collectively, the clinical data suggest that plasmid/virus- or plasmid/antigen-adjuvants, primeboost strategies generate strong immune responses and confirm the usefulness of DNA-based vaccination.

As tumor-associated antigens are usually poorly immunogenic altered self-molecules (e.g. melanocyte differentiation antigens), the induction of any immune response to these antigens involves breaking natural immune tolerance and may result in autoimmunity. As an example, several studies using xenogenic DNA immunization with DNA encoding the human tyrosinase have demonstrated tumor immunity as well as destruction of normal melanocytes with the clinical manifestation of vitiligo. ${ }^{48}$

\section{Topical spray application of liposomal DNA enables vaccination}

Although multiple approaches have been developed to deliver plasmid DNA to the skin, most gene transfer studies have focused on ex vivo approaches. In addition, several methods have been used successfully to deliver genes to the skin in vivo, including direct injection of naked DNA, gene gun, magnetofection, hydrodynamic injection or using an electric pulse. ${ }^{49}$ Polyplexes containing a variety of ligands (RGD peptide; anti-CD3; Fab antibody fragments; transferrin and galactose) coupled to polyethylenimine have achieved targeted endocytosis and have also resulted in gene delivery to keratinocytes in vitro. ${ }^{49}$ Generally, non-viral delivery relied on plasmid DNA and yielded transient expression between days and a few weeks.

Topical application of plasmid DNA represents an attractive route of gene delivery. If successful, it would have great prospects in transient skin gene therapy (e.g. DNA vaccination, wound healing, metabolic diseases etc.), since it is painless and easy to administer. Previous work has demonstrated that topically applied plasmids can access cellular targets via the hair follicles. Whether intercellular or transcellular routes of transportation play a role, has not yet been resolved. We have recently analyzed the expression of plasmid DNA in vivo and in vitro following topical application of plasmid DNA in nonionic liposomal spray formulations onto mouse or human skin using the enhanced green fluorescent protein. ${ }^{50}$ The results showed that EGFP mRNA and protein were detectable by RT-PCR and Western blot. However, EGFP protein was undetectable, when assessed by confocal laser microscopy or fluorescenceactivated cell sorting (FACS), in contrast to about $4 \%$ of fluorescent GFP positive keratinocytes detected after intradermal injection. ${ }^{50}$ In an in vivo mouse model the application of pEGFP-DNA led to the generation of GFPspecific antibodies. ${ }^{50}$ These results indicate that topical spray application of pEGFP liposomal DNA formulations is a suitable method for plasmid DNA delivery to the skin that may be useful for DNA vaccination. 
Extending previous results, we achieved sufficient penetration of plasmid DNA into the interfollicular epidermis of various strains of mice, including nude mice that lack anagen follicles, and human interfollicular skin. Given the abundance of interfollicular keratinocytes besides the scalp, axilla and groin, interfollicular uptake may even represent the principal route for human skin penetration.

\section{Mechanisms of DNA uptake and trafficking reveal fundamental insights into keratinocyte biology}

The successful development of topical skin gene therapy depends on a multi-step drug delivery process. ${ }^{50}$ The first step for DNA uptake following topical application involves the penetration of biologically active plasmid DNA through the stratum corneum. The stratum corneum, composed of dead epithelial cells and lipids, represents the principal barrier to penetration of macromolecules. Once this barrier is overcome, transport within the epidermis has to occur and plasmid DNA has to reach the keratinocyte cell membrane. Most likely, the liposomal composition and the concentration gradient play an important role in this regard. At the cell surface, uptake into the cytoplasm has to occur. When inside the cell, the majority of the plasmid will be degraded in endosomal and lysosomal compartments. However, a proportion of the DNA will be released from the acidic compartments and may eventually arrive at the nuclear membrane, where a fraction thereof may be shuffled into the nucleus for transcription to occur. All these steps will affect transfection efficiency in vivo.

Recently, we have characterized the in vitro uptake of plasmid DNA by keratinocytes in more detail showing that several forms of endocytosis (e.g. pinocytosis) seem to be involved. ${ }^{51}$ In this study, we attempted to visualize and measure uptake of naked FITC-labeled plasmid by FACS analysis detecting up to $15 \%$ internalization in a dose- and time-dependent manner. While oligodeoxynucleotides were rapidly and almost completely taken up into the nucleus of keratinocytes, only low amounts of FITC-labeled plasmid could be detected in the cytoplasm and around the nucleus after $6 \mathrm{~h}$ (Figure 4). The uptake depended on the concentration, time, temperature and on protein synthesis. ${ }^{51}$ Several established mechanisms of endocytosis, utilizing clathrin-coated pits, macropinocytosis and caveolae, were studied. FITC- and Cy5labeled plasmid DNA was applied together with specific inhibitors of macropinocytosis (amilorid and N,N-dimethylamilorid), potocytosis (nystatin and filipin) and clathrin-coated pits (chlorpromazine). ${ }^{51}$ These experiments suggested that macropinocytosis was the predominant mode of DNA uptake by keratinocytes.

When we analyzed intracellular trafficking of Cy5labeled plasmid DNA together with the 10-kDa FITCdextran, a commonly used fluid-phase endocytosis marker, co-localization was detected as soon as $8 \mathrm{~h}$ after incubation. ${ }^{51}$ Co-incubation with FITC-labeled transferrin as an indicator of receptor-mediated uptake and clathrin-coated pits showed no colocalization. When Cy5-labeled plasmid DNA was incubated together with the LysoTracker Green dye significant co-localization was detected in lysosomes at 24 h..$^{51}$
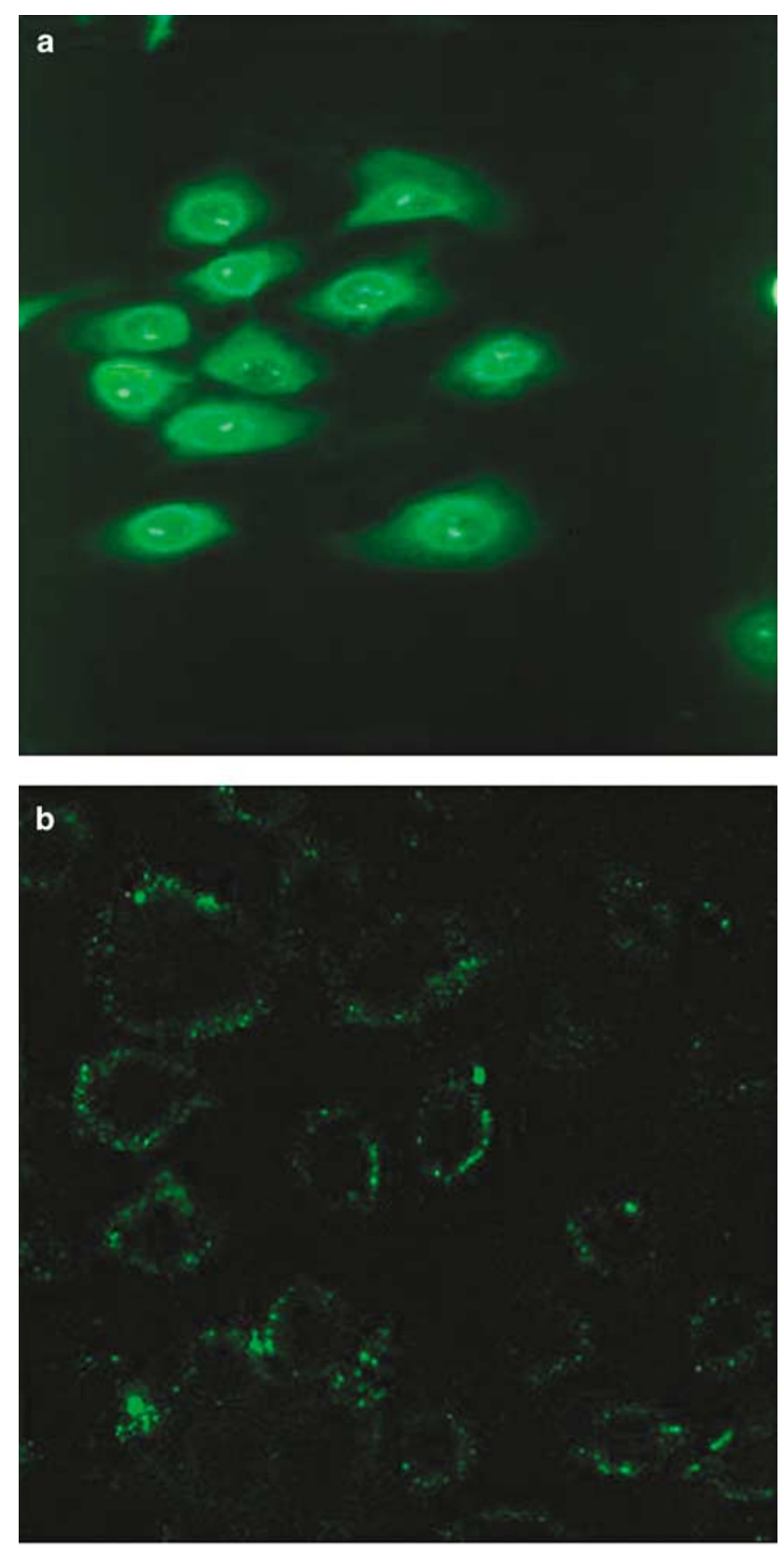

Figure 4 Visualization of plasmid uptake. Primary human keratinocytes were incubated in the presence of FITC-oligodeoxynucleotides (200mer; $4 \mu \mathrm{M}$ ) or naked FITC-labeled plasmid DNA $(1 \mu \mathrm{g} / \mathrm{ml})$. While oligodeoxy-nucleotides accumulated within $30 \mathrm{~min}$ and showed a nucloelar pattern (a), perinuclear accumulation of plasmid DNA was observed at $6 \mathrm{~h}(\mathbf{b})$. Original magnification $\times 630$. Taken from reference $\#^{51}$ with permission.

To detect potential DNA receptors on the keratinocyte surface, membrane proteins were extracted and subjected to South-Western blotting using digoxigeninlabeled calf thymus and lambda phage DNA. ${ }^{51}$ While we were not able to detect DNA-receptor proteins for uptake by receptor-mediated endocytosis, two DNAbinding proteins, ezrin and moesin were identified by South-Western blots and MALDI-mass spectrometry. Ezrin and moesin are known as plasma membrane-actin linkers, functionally associated with a number of transmembrane receptors such as the EGF-, CD44- or 
ICAM-1-receptor. Following ligand-binding to some of the associated receptors, phosphorylation of ezrin occurs at the C-terminus by a special protein kinase, leading to the resolution of head-to-tail ezrin binding and, thus, enabling the binding of actin. Within 2 min significant morphological changes occur such as the formation of microvilli, membrane ruffles and increased endocytosis.

Currently, we try to identify the DNA-binding domains within the ezrin molecule and the interactions of DNA with transmembrane receptors to further elucidate the biological function of this newly recognized property of proteins known as cytoskeleton-actin linkers.

\section{References}

1 Uitto J, Richard G. Progress in epidermolysis bullosa: genetic classification and clinical implications. Am J Med Genet C 2004; 131: $61-74$

2 Ortiz-Urda S, Lin Q, Green CL, Keene DR, Marinkovich MP, Khavari PA. Injection of genetically engineered fibroblasts corrects regenerated human epidermolysis bullosa skin tissue. J Clin Invest 2003; 111: 251-255.

3 Hengge UR, Bardenheuer W. Gene therapy and the skin. Am J Med Genet C 2004; 131: 93-100.

4 Gagnoux-Palacios L, Hervouet C, Spirito F, Roques S, Mezzina $\mathrm{M}$, Danos $\mathrm{O}$ et al. Assessment of optimal transduction of primary human skin keratinocytes by viral vectors. J Gene Med 2005; 7: 1178-1186.

5 Serrano F, Del Rio M, Larcher F, Garcia M, Munoz E, Escamez MJ et al. A comparison of targeting performance of oncoretroviral versus lentiviral vectors on human keratinocytes. Hum Gene Ther 2003; 14: 1579-1585.

6 Bajaj BG, Lei P, Andreadis ST. Efficient gene transfer to human epidermal keratinocytes on fibronectin: in vitro evidence for transduction of epidermal stem cells. Mol Ther 2005; 11: 969-979.

7 Braun-Falco M, Eisenried A, Buning H, Ring J. Recombinant adeno-associated virus type 2-mediated gene transfer into human keratinocytes is influenced by both the ubiquitin/ proteasome pathway and epidermal growth factor receptor tyrosine kinase. Arch Dermatol Res 2005; 296: 528-535.

$8 \mathrm{Lu} \mathrm{B}$, Makhija SK, Nettelbeck DM, Rivera AA, Wang M, Komarova $\mathrm{S}$ et al. Evaluation of tumor-specific promoter activities in melanoma. Gene Therapy 2005; 12: 330-338.

9 Okada Y, Okada N, Mizuguchi H, Hayakawa T, Nakagawa S, Mayumi T. Transcriptional targeting of RGD fiber-mutant adenovirus vectors can improve the safety of suicide gene therapy for murine melanoma. Cancer Gene Ther 2005; 12: 608-616.

10 Ortiz-Urda S, Lin Q, Yant SR, Keene D, Kay MA, Khavari PA. Sustainable correction of junctional epidermolysis bullosa via transposon-mediated nonviral gene transfer. Gene Therapy 2003; 10: 1099-1104.

11 Ortiz-Urda S, Thyagarajan B, Keene DR, Lin Q, Calos MP, Khavari PA. PhiC31 integrase-mediated nonviral genetic correction of junctional epidermolysis bullosa. Hum Gene Ther 2003; 14: 923-928.

12 Laposa RR, Feeney L, Cleaver JE. Recapitulation of the cellular xeroderma pigmentosum-variant phenotypes using short interfering for DNA polymerase H. Cancer Res 2003; 63: 3909-3912.

13 Urnov FD, Miller JC, Lee YL, Beausejour CM, Rock JM, Augustus $\mathrm{S}$ et al. Highly efficient endogenous human gene correction using designed zinc-finger nucleases. Nature 2005; 435: 646-651.

14 D'Alessandro M, Morley SM, Ogden PH, Liovic M, Porter RM, Lane EB. Functional improvement of mutant keratin cells on addition of desmin: an alternative approach to gene therapy for dominant diseases. Gene Therapy 2004; 11: 1290-1295.

15 Arin MJ, Roop DR. Inducible mouse models for inherited skin diseases: implications for skin gene therapy. Cells Tissues Organs 2004; 177: 160-168.

16 Baldeschi C, Gache Y, Rattenholl A, Bouille P, Danos O, Ortonne JP et al. Genetic correction of canine dystrophic epidermolysis bullosa mediated by retroviral vectors. Hum Mol Genet 2003; 12: 1897-1905.

17 Gache Y, Baldeschi C, Del Rio M, Gagnoux-Palacios L, Larcher F, Lacour JP, Meneguzzi G. Construction of skin equivalents for gene therapy of recessive dystrophic epidermolysis bullosa. Hum Gene Ther 2004; 15: 921-933.

18 Woodley DT, Keene DR, Atha T, Huang Y, Ram R, Kasahara N et al. Intradermal injection of lentiviral vectors corrects regenerated human dystrophic epidermolysis bullosa skin tissue in vivo. Mol Ther 2004; 10: 318-326.

19 Marchetto MC, Muotri AR, Burns DK, Friedberg EC, Menck CF. Gene transduction in skin cells: preventing cancer in xeroderma pigmentosum mice. Proc Natl Acad Sci USA 2004; 101: 17759-17764.

20 Zhang F, Yang F, Hu EC, Sones W, Lei M, Lineaweaver WC. Vascular endothelial growth factor gene therapy in improvement of skin paddle survival in a rat TRAM flap model. J Reconstr Microsurg 2005; 21: 391-396.

21 Carretero M, Del Rio M, Garcia M, Escamez MJ, Mirones I, Rivas $\mathrm{L}$ et al. A cutaneous gene therapy approach to treat infection through keratinocyte-targeted overexpression of antimicrobial peptides. FASEB J 2004; 18: 1931-1933.

22 Jacobsen F, Mittler D, Hirsch T, Gerhards A, Lehnhardt M, Voss $\mathrm{B}$ et al. Transient cutaneous adenoviral gene therapy with human host defense peptide hCAP-18/LL-37 is effective for the treatment of burn wound infections. Gene Therapy 2005; 12: 1494-1502.

23 Sawamura D, Goto M, Shibaki A, Akiyama M, McMillan JR, Abiko $\mathrm{Y}$ et al. Beta defensin-3 engineered epidermis shows highly protective effect for bacterial infection. Gene Therapy 2005; 12: 857-861.

24 Marti G, Ferguson M, Wang J, Byrnes C, Dieb R, Qaiser R et al. Electroporative transfection with KGF-1 DNA improves wound healing in a diabetic mouse model. Gene Therapy 2004; 11: $1780-1785$.

25 Lee PY, Chesnoy S, Huang L. Electroporatic delivery of TGFbeta1 gene works synergistically with electric therapy to enhance diabetic wound healing in $\mathrm{db} / \mathrm{db}$ mice. J Invest Dermatol 2004; 123: 791-798.

26 Liu W, Chua C, Wu X, Wang D, Ying D, Cui L et al. Inhibiting scar formation in rat wounds by adenovirus-mediated overexpression of truncated TGF-beta receptor II. Plast Reconstr Surg 2005; 115: 860-870.

27 Akiyama M, Sugiyama-Nakagiri Y, Sakai K, McMillan JR, Goto $\mathrm{M}$, Arita $\mathrm{K}$ et al. Mutations in lipid transporter ABCA12 in harlequin ichthyosis and functional recovery by corrective gene transfer. J Clin Invest 2005; 115: 1777-1784.

28 Lee H, Koehler DR, Pang CY, Levine RH, Ng P, Palmer DJ et al. Gene delivery to human sweat glands: a model for cystic fibrosis gene therapy. Gene Therapy 2005; 12: 1752-1760.

29 Heinzerling L, Burg G, Dummer R, Maier T, Oberholzer PA, Schultz J et al. Intratumoral injection of DNA encoding human interleukin 12 into patients with metastatic melanoma: clinical efficacy. Hum Gene Ther 2005; 16: 35-48.

30 Triozzi PL, Strong TV, Bucy RP, Allen KO, Carlisle RR, Moore SE et al. Intratumoral administration of a recombinant canarypox virus expressing interleukin 12 in patients with metastatic melanoma. Hum Gene Ther 2005; 16: 91-100.

31 Moiseyenko VM, Danilov AO, Baldueva IA, Danilova AB, Tyukavina NV, Larin SS et al. Phase I/II trial of gene therapy with autologous tumor cells modified with tag7/PGRP-S gene in 
patients with disseminated solid tumors: miscellaneous tumors. Ann Oncol 2005; 16: 162-168.

32 Meyer RG, Britten CM, Siepmann U, Petzold B, Sagban TA, Lehr HA et al. A phase I vaccination study with tyrosinase in patients with stage II melanoma using recombinant modified vaccinia virus Ankara (MVA-hTyr). Cancer Immunol Immunother 2005; 54: 453-467.

33 Schultz ES, Schuler-Thurner B, Stroobant V, Jenne L, Berger TG, Thielemanns $\mathrm{K}$ et al. Functional analysis of tumor-specific Th cell responses detected in melanoma patients after dendritic cell-based immunotherapy. J Immunol 2004; 172: 1304-1310.

34 Ghazizadeh S, Kalish RS, Taichman LB. Immune-mediated loss of transgene expression in skin: implications for cutaneous gene therapy. Mol Ther 2003; 7: 296-303.

$35 \mathrm{Lu} \mathrm{Z}$, Ghazizadeh S. Host immune responses in ex vivo approaches to cutaneous gene therapy targeted to keratinocytes. Exp Dermatol 2005; 14: 727-735.

36 Ohyama M, Ota T, Aoki M, Tsunoda K, Harada R, Koyasu S et al. Suppression of the immune response against exogenous desmoglein 3 in desmoglein 3 knockout mice: an implication for gene therapy. I Invest Dermatol 2003; 120: 610-615.

37 Mingozzi F, Liu YL, Dobrzynski E, Kaufhold A, Liu JH, Wang Y et al. Induction of immune tolerance to coagulation factor IX antigen by in vivo hepatic gene transfer. J Clin Invest 2003; 111: 1347-1356.

38 Hermans IF, Chong TW, Palmowski MJ, Harris AL, Cerundolo V. Synergistic effect of metronomic dosing of cyclophosphamide combined with specific antitumor immunotherapy in a murine melanoma model. Cancer Res 2003; 63: 8408-8413.

39 Dobrzynski E, Mingozzi F, Liu YL, Bendo E, Cao O, Wang L et al. Induction of antigen-specific CD4+ T-cell anergy and deletion by in vivo viral gene transfer. Blood 2004; 104: 969-977.

40 Gregor PD, Wolchok JD, Ferrone CR, Buchinshky H, GuevaraPatino JA, Perales MA et al. CTLA-4 blockade in combination with xenogeneic DNA vaccines enhances T-cell responses, tumor immunity and autoimmunity to self antigens in animal and cellular model systems. Vaccine 2004; 22: 1700-1708.
41 Prud'homme GJ. DNA vaccination against tumors. I Gene Med 2005; 7: 3-17.

42 Lisziewicz J, Trocio J, Whitman L, Varga G, Xu J, Bakare N et al. DermaVir: a novel topical vaccine for HIV/AIDS. I Invest Dermatol 2005; 124: 160-169.

43 Hess PR, Boczkowski D, Nair SK, Snyder D, Gilboa E. Vaccination with mRNAs encoding tumor-associated antigens and granulocyte-macrophage colony-stimulating factor efficiently primes CTL responses, but is insufficient to overcome tolerance to a model tumor/self antigen. Cancer Immunol Immunother 2005; 20: 1-12; (E-pub ahead of print).

44 Wang R, Epstein J, Charoenvit Y, Baraceros FM, Rahardjo N, Gay $\mathrm{T}$ et al. Induction in humans of CD8+ and CD4+ $\mathrm{T}$ cell and antibody responses by sequential immunization with malaria DNA and recombinant protein. J Immunol 2004; 172: 5561-5569.

45 McConkey SJ, Reece WH, Moorthy VS, Webster D, Dunachie S, Butcher $\mathrm{G}$ et al. Enhanced T-cell immunogenicity of plasmid DNA vaccines boosted by recombinant modified vaccinia virus Ankara in humans. Nat Med 2003; 9: 729-735.

46 Bramson J, Dayball K, Evelegh C, Wan YH, Page D, Smith A. Enabling topical immunization via microporation: a novel method for pain-free and needle-free delivery of adenovirusbased vaccines. Gene Therapy 2003; 10: 251-260.

47 Dayball K, Millar J, Miller M, Wan YH, Bramson J. Electroporation enables plasmid vaccines to elicit $\mathrm{CD} 8+\mathrm{T}$ cell responses in the absence of CD4+ T cells. J Immunol 2003; 171: 3379-3384.

48 Bergman PJ, McKnight J, Novosad A, Charney S, Farrelly J, Craft $\mathrm{D}$ et al. Long-term survival of dogs with advanced malignant melanoma after DNA vaccination with xenogeneic human tyrosinase: a phase I trial. Clin Cancer Res 2003; 9: 1284-1290.

49 Wolff JA, Budker V. The mechanism of naked DNA uptake and expression. Adv Genet 2005; 54: 3-20.

50 Meykadeh N, Mirmohammadsadegh A, Wang Z, BasnerTschakarjan E, Hengge UR. Topical application of plasmid DNA to mouse and human skin. J Mol Med 2005; 83: 897-903.

51 Basner-Tschakarjan E, Mirmohammadsadegh A, Baer A, Hengge UR. Uptake and trafficking of DNA in keratinocytes: evidence for DNA-binding proteins. Gene Therapy 2004; 11: 765-774. 